\title{
INDECISIÓN VOCACIONAL: PÉRDIDAS Y "PERDIDOS" EN LA EDUCACIÓN SUPERIOR
}

\author{
Arturo Prieto*
}

* Director de la Escuela de Ciencias Sociales de la Universidad de Viña del Mar. 


\section{RESUMEN}

La estandarización de la Escala Subjetiva de Intereses y Aptitudes Vocacionales reveló que uno de cada cuatro alumnos de $3^{\circ}$ y $4^{\circ}$ año de educación media presenta problemas vocacionales que, cuando ingrese a la Universidad, generarán pérdidas públicas y privadas. Nuestros jóvenes manifiestan tendencias vocacionales negativas de desagrado-inhabilidad en áreas ocupacionales relacionadas con la agricultura, defensa de la institucionalidad, industria, minería-geología y química, lo que a futuro podría implicar pérdidas para la actividad productiva, económica e institucional del país.

\section{ABSTRACT}

The standardization of the Subjective Scale of Interests and Vocational Aptitudes revealed that one out every four students in third and fourth grade in secondary education have vocational problems that, when they enroll in University, will give rise to private and public losses. Our young people manifest vocational trends of displeasure/inability in occupational areas related to agriculture, defense of the institutional framework, industry, mining-geology and chemistry and these facts could -in futureimply losses for the country's productive, economic and institutional activities. 


\section{INDECISIÓN VOCACIONAL: PÉRDIDAS Y "PERDIDOS" EN LA EDUCACIÓN SUPERIOR}

La investigación ha revelado que la indecisión vocacional está asociada, por una parte, a problemas clínicos y psicopatológicos y, por otra, a problemas socioeconómicos producidos por la pérdida de:

a) tiempo de un joven que estudia una carrera que no satisface sus necesidades personales;

b) dinero en la familia que invierte en educación superior inconclusa;

c) recursos académicos por efecto de la deserción y movilidad estudiantil en la educación superior;

d) recursos del Estado, al postergar la inserción productiva de los ciudadanos en la sociedad.

De los resultados del trabajo de estandarización de la Escala Subjetiva de Intereses y Aptitudes Vocacionales (ESIAV) -investigación sobre la cual se sustenta el presente artículo- es posible inferir que un bajo rendimiento académico, así como la deserción y movilidad de los estudiantes en el sistema universitario, parecen estar más relacionados con sus problemas vocacionales que con déficit cognitivos.

La decisión de un joven acerca de la alternativa profesional que resulta más compatible con su proyecto de vida involucra la conjunción de procesos sociales, psicológicos e incluso biológicos (Luzzo, 2000; Luzzo y MacGregor, 2000; Lent, Brown y Hackett, 1994), que determinarán su desarrollo personal, calidad de vida y contribución a la sociedad (Paa y McWhirter, 2000; Prieto, 1999 y 2002). En general y a modo de síntesis, las líneas de investigación vocacional actualmente predominantes sugieren que los procesos que determinan la decisión profesional pueden agruparse en cuatro factores que inciden en la realización de actividades ocupacionales: 
$1^{\circ}$ Intereses (Lapan, Adams, Turner y Hinkelman, 2000; Lapan, Hinkelman, Adams y Turner, 1999; Prediger, 1996; Crites, 1974) que incluyen las características personales, físicas y psicológicas, tales como la salud y constitución corporal, temperamento, rasgos de personalidad (Holland, 1999; Luzzo y MacGregor, 2000), preferencias, motivaciones (Asner-Self, 2001; Lapan, Hinkelman, Adams y Turner, 1999; Crites, 1974) y afectos.

$2^{\circ}$ Aptitudes (Asner-Self, 2001; Prediger, 1996) que involucran la capacidad intelectual, general y específica (Asner-Self, 2001; Arbona, 1999); coordinaciones sensoperceptuales y motrices, destrezas y talentos especiales; procesos cognitivos (Lent, Brown y Hackett, 1994; Luzzo y MacGregor, 2000; Heppner, O’Brien, Hinkelman y Humphrey, 1994) y expectativas de autoeficacia (Ferry, Fouad y Smith, 2000; Luzzo y MacGregor, 2000; Betz, Schifano y Kaplan, 1999; Lapan, Hinkelman, Adams y Turner, 1999; Heppner, O’Brien y Hinkelman, 1996; Bandura, 1989).

$3^{\circ}$ Valores sociales (Lapan, Hinkelman, Adams y Turner, 1999) que implican el juicio moral y ético; opinión pública, política, económica y social; disposiciones actitudinales (Asner-Self, 2001; Heppner, O’Brien y Hinkelman, 1996) y creencias religiosas.

$4^{\circ}$ Ambiente (Holland, 1997; Lapan, Hinkelman, Adams y Turner, 1999; Swanson y Fouad, 1999; Hinkelman y Luzzo, 1997) referido al tipo de vida urbana o rural; disponibilidad y expectativas de campo laboral (Hinkelman, 2000; Lapan, Hinkelman, Adams y Turner, 1999); presencia de modelos significativos relacionados con las alternativas ocupacionales disponibles; barreras y/o facilitadores familiares (Weihs, Fisher y Baird, 2002), económicos, estatales y socioculturales; oportunidad educacional (Santos, Ferreira y Chaves, 2001; Swanson y Fouad, 1999).

En contraste con estas evidencias, los instrumentos de evaluación vocacional existentes hasta la fecha medían los intereses, aptitudes, valores y ambiente mediante métodos independientes entre sí (por ejemplo: Knapp-Lee, 2000; Harmon, Hansen, Borgen y Hammer, 1994; Osipow, Carney, Winer, Yanico y Koschier, 1987; Super, Thompson, Lindeman, Jordaan y Myers, 1981; Crites, 1978), los que comúnmente 
son agrupados en grandes categorías: arte, ciencia, tecnología, humanidades (Rounds y Day, 1999; Prediger, 1982 y 1996). No obstante ahora, con la estandarización de la ESIAV es posible medir y comparar los intereses, aptitudes y vocaciones con el mismo instrumento en un rango diferenciado de 22 áreas ocupacionales. Este nuevo instrumento vocacional permite establecer la(s) carrera(s) profesional(es) que se ajusta(n) mejor a las características ideográficas de cada alumno de enseñanza media (EM). A su vez, en la educación superior permitiría orientar y predecir el nivel de éxito, realización personal y continuidad de formación que obtendrá el estudiante en la carrera que eligió, reduciendo con ello la deserción y movilidad estudiantil. Para efectos de mejorar la retención en carreras universitarias parece recomendable entonces que el sistema de ingreso a la educación superior -más allá de los requerimientos académicos, cognitivos y de competencias personales de una determinada carrerapudiese contemplar una evaluación de las tendencias vocacionales compatibles con el perfil profesional de la(s) carrera(s) a la(s) que se postula. El presente artículo aborda las características psicométricas de la ESIAV y los resultados obtenidos con alumnos de EM, que permiten inferir la incidencia de los problemas vocacionales en la población estudiantil universitaria.

\section{ESCALA SUBJETIVA DE INTERESES Y APTITUDES VOCACIONALES}

Las características del método de evaluación de la ESIAV (Prieto, 2002 y 1999) son principalmente las siguientes:

$1^{\circ}$ Las actividades descritas en cada ítem son neutrales, es decir, sin una valoración previa;

$2^{\circ}$ Está compuesto por 22 áreas, cada una con 10 ítemes previamente validados por expertos (Cohen y Swerdlik, 2001; Asner-Self, 2001);

$3^{\circ}$ Las áreas de actividades ocupacionales evaluadas en el instrumento son: acciones administrativas, agricultura, artes plástico-gráficas, biología, clínica, construcción, defensa de la institucionalidad, deportes, electricidad-electrónica, empresa, física, industria, informática, literatura, matemáticas, mecánica, minería-geología, música, pedagogía, química, social y veterinaria; 
$4^{\circ}$ Las escalas de interés (I) y aptitud (A) se aplican por separado, en ese orden, y consecutivamente a todo el cuestionario;

$5^{\circ}$ La escala de vocación (V) en cada área corresponde a la suma de las puntuaciones de I y A, las que pueden ser positivas o negativas;

$6^{\circ}$ Tomando el total de las puntuaciones parciales de I, A y V a través de todas las áreas, se obtienen variables generales e indicadores de consistencia que describen procesos globales del desarrollo y estructura vocacional del examinado.

Un ejemplo de ítem neutral sería: Ofrecer para venta un producto nuevo a personas que no conozco. Se autoevalúa cada ítem mediante escalas Likert diferentes para I y A, pero que son equivalentes y comparables entre sí. En la escala de interés se pide al sujeto que autoevalúe el nivel de agrado-desagrado que le produciría realizar cada actividad ocupacional, marcando una de las cinco categorías: a) me agrada mucho; b) me agrada; c) me es indiferente; d) me desagrada; e) me desagrada mucho. La escala de aptitud se aplica de un modo análogo a la de los intereses; se pide ahora al sujeto que juzgue en un continuo de habilidad-inhabilidad, qué tan bueno o malo se considera él para realizar esas mismas actividades: a) muy bueno; b) bueno; c) regular; d) malo; e) muy malo. Para ambas escalas se asignan los siguientes valores a cada alternativa $a=2 ; b=1 ; c=0 ; d=-1$ y $e=-2$ puntos. De la suma de los puntos de los 10 ítemes de cada área, se obtiene una puntuación independiente pero equivalente y comparable entre I y A en esas áreas. De la suma entre las puntuaciones de I y A en cada área se obtiene la escala vocación para esa área. Por ejemplo, si en la escala I un sujeto dijera que le agrada mucho explorar nuevos softwares, y en la escala A dijese que es bueno para examinar softwares, entonces él presenta consistencia positiva en el área informática. Si dijese en cambio, que le agrada tocar guitarra, pero es muy malo para eso, entonces es inconsistente en el área música. Pero si declara que le desagrada mucho vender, y además que es muy malo para vender, entonces él presenta consistencia negativa para los negocios.

En la ESIAV la vocación es definida como la consistencia positiva o negativa entre la autopercepción de I y A en un área ocupacional, de manera que se distinguen dos tipos de vocación:

Vocación positiva: está dada por la consistencia de la autovaloración entre las dimensiones de agrado-habilidad, respectivas al interés y aptitud en cada área ocupacional. 
Vocación negativa: está dada por la consistencia de la autovaloración entre las dimensiones de desagrado-inhabilidad, relativas al interés y aptitud en cada área ocupacional.

Asimismo, entre nueve categorías de (in)consistencia, pueden diferenciarse dos formas típicas de inconsistencia, según cuál sea la combinación positiva y negativa entre el interés y la aptitud autopercibida en cada área ocupacional:

Inconsistencia -I;+A: está dada por la combinación entre las dimensiones de desagrado-habilidad de la autovaloración, relativas al interés y aptitud en cada área ocupacional.

Inconsistencia $+\mathrm{I} ;-\mathbf{A}$ : está dada por la combinación entre las dimensiones de agrado-inhabilidad de la autovaloración, relativas al interés y aptitud en cada área ocupacional.

En cambio, cuando la combinación de las escalas de I y A son respectivamente consistentes con la autovaloración indiferente-regular de las actividades ocupacionales representativas de un área, entonces corresponde propiamente a la ausencia de vocación en esa área ocupacional. Todas estas combinaciones entre las dimensiones de I y A en cada área permiten obtener el perfil y jerarquía vocacional del examinado, lo que junto con las puntuaciones de las variables generales e indicadores de consistencia, que se calculan desde el conjunto de puntuaciones parciales de todas las áreas ocupacionales, constituyen información relevante acerca de la estructura, dinámica y desarrollo vocacional del examinado, solamente cuando sus puntuaciones en cada área y variables generales puedan ser contrastadas con los estadísticos paramétricos y baremos correspondientes a su grupo "etario" y de género.

\section{MÉTODO}

Se aplicó la ESIAV para obtener las normas de estandarización y analizar las características vocacionales en una muestra de 509 alumnos de EM. Debido a las diferencias de género relativas a expectativas ocupacionales, intereses y aptitudes (Lapan, Adams, Turner y Hinkelman, 2000), se diferenciaron dos grupos: 236 hombres y 273 
mujeres. Todos los alumnos, al momento de la evaluación, estaban cursando el $3^{\circ}$ o el $4^{\circ}$ año de EM. No se incluyeron sujetos con estudios universitarios para evitar la interferencia de sus decisiones vocacionales previas. Al momento de la evaluación, los alumnos tenían entre 16 y 18 años de edad. Dado que el tipo de colegio, si es privado o público, es también una variable que influye en las preferencias vocacionales de los adolescentes (Mau y Bikos, 2000), la procedencia de los sujetos correspondió a cuatro colegios particulares y cuatro establecimientos educacionales municipalizados, todos de la V Región.

En el análisis de datos, diferenciados por género (Cohen y Swerdlik, 2001; Pagano, 1999; Runyon y Haber, 1992; Garrett, 1990; Glass y Stanley, 1986), se obtuvieron: la media, desviación estándar, percentil, rango percentil, mediana, cuartil y prueba $Z$ para cada área profesional en las escalas de interés, aptitud y vocación; para las variables generales en las escalas de I, A y V, y para los indicadores de consistencia en el nivel global y por áreas.

\section{RESULTADOS Y DISCUSIÓN}

Los estadísticos paramétricos de todas las variables analizadas en hombres y mujeres se encuentran en el informe de estandarización (Prieto, 2002). Aunque los resultados revelan perfiles vocacionales significativamente discriminantes de género, para efectos del presente artículo se mostrarán las tendencias generales, de hombres y mujeres, que incidirían sobre pérdidas privadas y públicas derivadas de los problemas vocacionales.

En la tabla 1 se exponen las jerarquías vocacionales para hombres y mujeres. Las áreas son presentadas numéricamente de mayor a menor; en letra normal las que son vocaciones positivas y en cursiva las negativas; aquellas áreas cuyas medias son distintivas de cada sexo con una significación de $\mu=0.05$, en mayúsculas negritas. 
Experiencias para incrementar la retención en la educación superior. Diversos enfoques

Tabla 1:

Jerarquías vocacionales de hombres y mujeres

\begin{tabular}{|c|c|c|c|}
\hline Jerarquía vocacional hombres & Medias & Jerarquía vocacional mujeres & Medias \\
\hline INFORMÁTICA & 11.17 & CLÍNICA & 15.08 \\
\hline ELECTRICIDAD-ELECTRÓNICA & 5.80 & SOCIAL & 10.33 \\
\hline Deportes & 4.95 & ARTES PLÁSTICO-GRÁFICAS & 6.32 \\
\hline Clínica & 4.14 & PEDAGOGÍA & 4.43 \\
\hline MECÁNICA & 3.79 & LITERATURA & 4.33 \\
\hline CONSTRUCCIÓN & 3.32 & BIOLOGÍA & 3.23 \\
\hline Música & 2.50 & Deportes & 2.52 \\
\hline Social & 2.30 & Acciones administrativas & 2.52 \\
\hline EMPRESA & 1.59 & VETERINARIA & 2.04 \\
\hline Artes plástico-gráficas & 1.14 & Música & -0.41 \\
\hline FÍSICA & 1.01 & Agricultura & -1.05 \\
\hline Acciones administrativas & 0.50 & Empresa & -3.85 \\
\hline MATEMÁTICAS & -0.24 & Informática & -5.15 \\
\hline INDUSTRIA & -0.26 & Defensa de la institucionalidad & -5.85 \\
\hline Veterinaria & -1.45 & Construcción & -6.17 \\
\hline MINERÍA-GEOLOGÍA & -1.75 & Industria & -6.39 \\
\hline Biología & -2.02 & Química & -7.70 \\
\hline Pedagogía & -2.18 & Minería-geología & -9.62 \\
\hline Defensa de la institucionalidad & -3.06 & Matemáticas & -11.08 \\
\hline Agricultura & -3.47 & Física & -11.20 \\
\hline Literatura & -5.50 & Electricidad-electrónica & -12.99 \\
\hline Química & -6.03 & Mecánica & -13.16 \\
\hline
\end{tabular}

En la tabla 1 la magnitud de las vocaciones positivas está en orden descendente, mientras que la magnitud absoluta de las negativas está en orden ascendente. Así, las áreas que ocupan el extremo superior del cuadro corresponden a las más altas vocaciones positivas; en cambio, las áreas que representan las más altas vocaciones negativas se ubican en el extremo inferior, lo que refleja el carácter bidimensional de las vocaciones.

Un aspecto de estos resultados, que reviste implicaciones de relevancia para el desarrollo y funcionamiento institucional de un país, es contar con la información proporcionada por normas nacionales acerca de cuál y cuánta es la tendencia vocacional de la población joven hacia las áreas ocupacionales de trascendencia para el fomento 
de la producción, economía e institucionalidad del país. Como se desprende de la tabla 1, las áreas que son valoradas como negativas en hombres y mujeres, y que revisten la mayor relevancia para el desarrollo de un país son: agricultura, defensa de la institucionalidad, industria, minería-geología y química. Si las tendencias vocacionales de esta muestra de alumnos de EM hacia las áreas que inciden directamente en el desarrollo del país son, en promedio, valoradas como actividades ocupacionales negativas y ante las cuales los jóvenes experimentan "desagrado e inhabilidad", lamentablemente entonces el desarrollo futuro de esas áreas en Chile puede verse limitado por la menor demanda vocacional hacia ellas. Este hecho también presagiaría indirectamente un oscuro porvenir para el desarrollo nacional de la agricultura, industria y minería, actividades que actualmente sustentan la producción y economía chilena. La negativa valoración de los jóvenes hacia el área defensa de la institucionalidad puede explicar el característico "desagrado e inhabilidad" que ellos manifiestan hacia las funciones y actividades cívicas; refleja también, sus tendencias negativas hacia la identidad histórico-institucional del país. Si bien estas conclusiones deben ser interpretadas como una "fotografía" actual de la realidad vocacional de los jóvenes de la V Región, es probable que se observen tendencias diferentes en otras regiones de Chile, por lo que parece recomendable replicar este estudio con jóvenes del norte, centro y sur del país.

La actual oferta de carreras profesionales en la educación superior chilena parece responder adecuadamente a las tendencias vocacionales de los jóvenes de hoy, quienes presentan en su mayoría preferencias compatibles con las alternativas profesionales orientadas a servicios, negocios, artes, ciencia y tecnología. Sin embargo, queda la duda si esta oferta universitaria actual podrá responder satisfactoriamente a las necesidades futuras del desarrollo de la producción, economía e institucionalidad del país. No vaya a suceder en un futuro mediato, que el actual furor por la formación de profesionales en las áreas de servicios, ciencias o artes, por ejemplo, deje al país sin una suficiente proporción de población que se ocupe de la producción industrial, agrícola o minera.

Presumiblemente, una medida para prevenir una futura pérdida de la capacidad productiva del país sea, por un lado, la evaluación 
vocacional de los jóvenes que hoy presentan intereses y aptitudes hacia actividades ocupacionales que podrían orientarse a la educación técnica aplicada a los procesos productivos; por otro lado, mediante incentivos públicos y oportunidades estatales, cabría promover el desarrollo de vocaciones prácticas aplicadas a las áreas de producción.

Los resultados del análisis de las variables generales y consistencia de la ESIAV sugieren diferencias significativas en la estructura vocacional atribuibles al género, relativas a la forma en que hombres y mujeres enfrentan el mundo profesional (Perrone, Sedlacek y Alexander, 2001). Si bien no se observaron diferencias atribuibles al género entre las categorías de (in)consistencia, ya que tanto hombres como mujeres son más consistentes que inconsistentes, los resultados generales revelan que el $25 \%$ de los jóvenes presenta inconsistencia vocacional que puede implicar riesgos clínicos ante diferentes trastornos psicopatológicos.

La alta incidencia de problemas vocacionales, en donde uno de cada cuatro alumnos de EM presenta inconsistencia de riesgo clínico, sugiere que algo similar debe ocurrir entre los estudiantes universitarios. Sobre este escenario, los problemas de retención, deserción y movilidad en la educación superior podrían provenir de dos diferentes fuentes. En primer lugar, la investigación psicológica revela que la presencia de interés por una carrera profesional genera un incremento de las expectativas de éxito del estudiante sobre su rendimiento académico (Brown, Lent y Gore, 2000; Gore y Leuwerke, 2000; Prediger, 1996 y 1999), en tanto que intereses negativos por la carrera están asociados a falta de expectativas de autoeficacia y bajo desempeño académico, generando sentimientos de fracaso y frustración vocacional (Lent, Brown y Hackett, 1994 y 2000). De este modo, los problemas de rendimiento académico que se asumen, pueden causar la deserción y abandono en la educación superior; parecen estar más relacionados con dificultades vocacionales que con una supuesta baja "aptitud académica" de los estudiantes. Asimismo, es posible que en algunos casos, puntajes bajos en la PAA puedan reflejar más bien problemas de indefinición vocacional o falta de interés por carreras universitarias, que de "ineptitud académica" atribuible a un déficit cognitivo del alumno. 
Las pérdidas privadas y públicas asociadas a las dificultades vocacionales son un problema que hasta la fecha no se ha cuantificado; no obstante, es presumible que esas pérdidas representen cifras relevantes para el estudiante, su familia y el Estado. Si consideramos que las dificultades vocacionales pueden extenderse en promedio durante dos años de la vida de un joven, y que durante esos dos años el estudiante universitario ocupa recursos académicos en la educación superior, gasta recursos financieros correspondientes a los aranceles de dos años de formación universitaria inconclusa y se retrasará además dos años por lo menos en incorporarse a la sociedad en forma productiva, entonces las dificultades vocacionales debieran ser consideradas como un problema de país, en la medida en que su incidencia en la población provoca significativas pérdidas al desarrollo humano, económico e institucional. En la realidad de la educación superior chilena, la orientación vocacional, lejos de ser abordada por políticas públicas ante una necesidad educacional de la población, ha sido relegada al plano de lo individual y privado de cada joven chileno, dejando la decisión vocacional en manos del ensayo y el error. Los intentos de las universidades chilenas por reducir las pérdidas provocadas por la movilidad, deserción y abandono estudiantil parecen "palos de ciego", entre los cuales pueden encontrarse algunos sistemas de nivelación académica "remediales" y programas curriculares generales. Estos últimos pretenden disfrazar la indecisión vocacional de algunos estudiantes ofreciendo una amplia y flexible malla de estudios que promete diversas "salidas" profesionales, como si fuese suficiente que con planes generales los estudiantes consigan salvar las barreras educacionales para tomar su decisión por algunas de esas salidas profesionales.

Tal vez la desesperanza de los "perdidos" en la educación superior y las debilidades de las políticas públicas en torno de los problemas vocacionales, puedan deberse al uso de instrumentos vocacionales antiguos, ineficaces y poco representativos de la población chilena contemporánea. Los instrumentos vocacionales usados en nuestro país tienen más de tres décadas de antigüedad y simplemente ya no reflejan las alternativas ocupacionales de la sociedad moderna y, menos aún, las tendencias vocacionales de la juventud de hoy. Además, los modelos evaluativos de esos instrumentos están atrasados respecto de las evidencias recogidas de la investigación vocacional actual. Por todo, 
resulta comprensible que los problemas vocacionales en nuestro país generen semejantes pérdidas y "perdidos" en la educación superior. Y no es mucho lo que se solucionará con sistemas de nivelación "remediales" o con programas curriculares generales para reducir la movilidad, deserción y abandono en estudiantes universitarios, mientras no se les brinde la oportunidad de tomar sus decisiones profesionales fundadas en un instrumento vocacional válido, moderno, eficaz y estandarizado para nuestra población juvenil.

La segunda fuente que puede producir considerables pérdidas en la educación superior es el alto riesgo de problemas clínicos en la población estudiantil universitaria, asociado a los problemas vocacionales (González, Tinsley y Kreuder, 2002; Pistole y Roberts, 2002). Contundentes evidencias de la investigación señalan que la indecisión vocacional está relacionada con trastornos clínicos y psicopatológicos (Anderson y Niles, 2000; Lindley y Borgen, 2000; Luzzo y MacGregor, 2000; Saunders, Peterson, Sampson y Reardon, 2000), la que de ser detectada oportunamente permitiría intervenir en forma preventiva en ese $25 \%$ de la población juvenil que presenta inconsistencia vocacional. Por ejemplo, un déficit común entre los problemas de drogodependencia y los vocacionales es la dificultad para tomar decisiones, que se refleja en la imposibilidad de decidirno-consumir drogas y/o en la indecisión vocacional. Los indicadores de inconsistencia de la ESIAV permiten establecer un prediagnóstico de la población juvenil, detectando aquellos sujetos que tienen altos indicadores de inconsistencia y por ende presentarían también un mayor riesgo de drogodependencia. Una evaluación vocacional oportuna podría ser la clave para una intervención preventiva en estudiantes de EM y universitarios, antes de que se bloquee la capacidad decisional frente al consumo de drogas u otras patologías psicológicas.

Ya sea desde su neta utilidad en el diagnóstico y consejería vocacional; desde las aplicaciones educacionales a nivel de EM y reducción de la deserción y movilidad estudiantil universitaria; desde sus posibles utilidades laborales referidas a la "ubicación ocupacional" de jóvenes desempleados; desde las implicancias comunitarias para proponer al Estado estrategias y políticas públicas relativas a la inserción laboral e incentivos que motiven a los jóvenes a capacitarse en áreas cruciales para la producción, economía e institucionalidad 
del país; o bien, desde las aplicaciones en la intervención clínica, la ESIAV constituye un instrumento de apoyo que, a través de información relevante y significativa para el proceso de decisión profesional, mejora la calidad de vida y propaga las potencialidades de transformación evolutiva del ser humano, que le permitirán alcanzar el éxito y la realización personal. Estas posibles aplicaciones, que cruzan diversos ámbitos de las ciencias sociales, son la contribución de este instrumento de evaluación vocacional a la expansión del desarrollo humano. La ESIAV constituye un intento para satisfacer la necesidad y el derecho de la juventud actual de tener una moderna y eficaz consejería vocacional. 


\section{REFERENCIAS BibliográFicas y Notas}

Anderson, W. P. Jr. y Niles, S. G., "Important events in career counseling: Client and counselor descriptions"; en The Career Development Quarterly, 48, 2000, pp. 251-263.

Arbona, C., "Practice and research in career counseling and development-1999"; en The Career Development Quarterly, 49, 1999, pp. 98-134.

Asner-Self, K. K., "Principles and application of assessment in counseling", revisión de S. C. Whiston (2000). "Principles and application of assessment in counseling"; en Measurement and Evaluation in Counseling and Development, 33, 2001, pp. 244-248.

Bandura, A., "Human agency in social cognitive theory"; en American Psychologist, 44, 1989, pp. 1175-1184.

Betz, N., Schifano, R. y Kaplan, A., "Relationships among measures of perceived self-efficacy with respect to basic domains of vocational activity"; en Journal of Career Assessment, 7, 1999, p. 213.

Brown, S. D., Lent, R. W. y Gore, P. A., "Self-rated abilities and selfefficacy beliefs: Are they empirically distinct?"; en Journal of Career Assessment, 8, 2000, pp. 223-235.

Cohen, R. J. y Swerdlik, M. E., Pruebas y evaluación psicológicas. Introducción a las pruebas y a la medición (4ª edición), McGraw-Hill, México, 2001.

Crites, J. O., Psicología vocacional, Paidós, Buenos Aires, Argentina, 1974.

Crites, J. O., The Career Maturity Inventory, CA: CTB/McGraw Hill, Monterrey, México, 1978.

Ferry, T. R., Fouad, N. A. y Smith, P. L., "The role of family context in a social cognitive model for career-related choice and behavior: A math 
and science perspective"; en Journal of Vocational Behavior, 57, 2000, pp. 348-364.

Garrett, H. E., Estadística en psicología y educación, Paidós, México, 1990.

Glass, G. V. y Stanley, J. C., Métodos estadísticos aplicados a las ciencias sociales, Prentice-Hall Hispanoamericana, México, 1986.

González, J. M., Tinsley, H. E. y Kreuder, K. R., "Effects of psychoeducational interventions on opinions of mental illness, attitudes toward help seeking, and expectations about psychotherapy in college students; en Journal of College Student Development, 2002, 43 , p. 51.

Gore, P. A. y Leuwerke, W. C., "Predicting occupational considerations: A comparison of self-efficacy beliefs, outcome expectations, and person-environment congruence"; en Journal of Career Assessment, 8, 2000, pp. 237-250.

Harmon, L. W., Hansen, J. C., Borgen, F. H. y Hammer, A. L., Strong Interest Inventory: Applications and technical guide, CA: Consulting Psychologists Press, Palo Alto, EE.UU., 1994.

Heppner, M. J., O'Brien, K. M. y Hinkelman, J. M., "Training counseling psychologists in career development: Are we our worst enemies?"; en The Counseling Psychologist, 24, 1996, pp. 105-125.

Heppner, M. J., O’Brien, K. M., Hinkelman, J. M. y Humphrey, C. F., "Shifting the paradigm: The use of creativity in career counseling"; en Journal of Career Development, 21, 1994, pp. 77-86.

Hinkelman, J. M., "Preparing students for the global marketplace"; en Career Planning and Adult Development Journal, 16, 2000, pp. 117122.

Hinkelman, J. M. y Luzzo, D. A., "Computer-assisted career guidance: bridging the science-practitioner gap"; en Career Planning and Adult Development Journal, 13, 1997, pp. 41-51. 
Holland, J. L., Making vocational choices ( $3^{a}$ edición), Psychological Assessment Resources, Odessa, FL; EE.UU., 1997.

Holland, J. L., "Why interest inventories are also personality inventories", en M. L. Savickas y A. RL Spokane (Eds.), Vocational interests meaning, measurement, and counseling use: Davies-Black, Palo Alto, CA, EE.UU., 1999.

Knapp-Lee, L., "A complete career guidance program: The COPSystem"; en C. E. Watkins y V. L. Campbell (Eds.), Testing and assessment in counseling practice ( $2^{a}$ edición), Lawrence Erlbaum Associates, Mahwah, NJ, EE.UU., 2000.

Lapan, R. T., Adams, A., Turner, S. y Hinkelman, J. M., "Seventh grader's vocational interest and efficacy expectation patterns"; en Journal of Career Development, 26, 2000, pp. 215-229.

Lapan, R. T., Hinkelman, J. M., Adams, A. y Turner, S., "Understanding rural adolescents interests, values, and efficacy expectations"; en Journal of Career Development, 26, 1999, pp. 107-124.

Lent, R. W., Brown, S. D. y Hackett, G., "Toward a unifying social cognitive theory of career and academic interest, choice, and performance"; en Journal of Vocational Behavior, 45, 1994, pp. 79-122.

Lent, R. W., Brown, S. D. y Hackett, G., "Contextual supports and barriers to career choice: A social cognitive analysis"; en Journal of Counseling Psychology, 47, 2000, pp. 36-49.

Lindley, L. D. y Borgen, F. H., "Personal style scales of the Strong Interest Inventory: Linking personality and interests"; en Journal of Vocational Behavior, 57, 2000, pp. 22-41.

Luzzo, D. A. (Ed.), Career counseling of college students: An empirical guide to strategies that work. American Psychological Association, Washington DC, EE.UU., 2000.

Luzzo, D. A. y MacGregor, M., "Practice and research in career counseling and development"; en The Career Development Quarterly, 50, 2000, pp. 98-139. 
Mau, W. y Bikos, L. H., "Educational and vocational aspirations of minority and female students: A longitudinal study"; en Journal of Counseling and Development, 78, 2000, pp. 186-194.

Osipow, S. H., Carney, C. G., Winer, J. L., Yanico, B. y Koschier, M., The Career Decision Scale (3 ${ }^{a}$ revisión), Psychological Assessment Resources, Odessa, FL, EE.UU., 1987.

Paa, H. K. y McWhirter, E. H., "Perceived influences on high school student's current career expectations"; en The Career Development Quarterly, 49, 2000, pp. 29-44.

Pagano, R. R., Estadística para las ciencias del comportamiento ( $5^{\mathrm{a}}$ edición), International Thompson, México, 1999.

Perrone, K. M., Sedlacek, W. E. y Alexander, C. M., "Gender and ethnic differences in career goal attainment"; en The Career Development Quarterly, 50, 2001, pp. 168-178.

Pistole, M. C. y Roberts, A.,"Mental health counseling: Toward resolving identity confutions"; en Journal of Mental Health Counseling, 24, 2002, pp. 1-19.

Prediger, D. J., "Dimensions underlying Holland's hexagon: Missing link between interests and occupations"; en Journal of Vocational Behavior, 21, 1982, pp. 259-287.

Prediger, D. J. "Integrating interests and abilities for career exploration: General considerations"; en M. L. Savickas y A. R. Spokane (Eds.), Vocational interests: Meaning, measurement, and counseling use, DaviesBlack, Palo Alto, CA, EE.UU., 1996.

Prediger, D. J., "Is interest profile level relevant to career counseling?"; en Journal of Counseling Psychology, 45, 1998, pp. 204-211.

Prediger, D. J., "Basic structure of work-relevant abilities"; en Journal of Counseling Psychology, 46, 1999, pp. 173-184.

Prieto, A., Escala subjetiva de intereses y aptitudes vocacionales. Manual de administración, Educactiva, Viña del Mar, Chile, 1999. 
Prieto, A., Estandarización de la escala subjetiva de intereses y aptitudes vocacionales; informe de investigación doctoral, Facultad de Psicología, Universidad de Granada, España, 2002.

Rounds, J. y Day, S. X., "Describing, evaluating, and creating vocational interest structures"; en M. L. Savickas y A. R Spokane (Eds.), Vocational interests: Meaning, measurement, and counseling use, Davies-Black, Palo Alto, CA, EE.UU., 1999.

Runyon, R. P. y Haber, A., Estadística para las ciencias sociales, AddisonWesley Iberoamericana, Wilmington, EE.UU., 1992.

Santos, E. J., Ferreira, J. A. y Chaves, A., "Implications of sociopolitical context for career services delivery"; en The Career Development Quarterly, 50, 2001, pp. 45-55.

Saunders, D. E., Peterson, G. W., Sampson, J. P. Jr. y Reardon, R. C., "Relation of depression and dysfunctional career thinking to career indecision"; en Journal of Vocational Behavior, 56, 2000, pp. 228-298.

Super, D. E., Thompson, A. S., Lindeman, R. H., Jordaan, J. P. y Myers, R. A., The Career Development Inventory, Consulting Psychologists Press, Palo Alto, CA, EE.UU., 1981.

Swanson, J. L. y Fouad, N. A., "Applying theories of personenvironment fit to the transition from school to work"; en The Career Development Quarterly, 47, 1999, pp. 337-347.

Weihs, K., Fisher, L. y Baird, M., "Families, health, and behavior"; en Families, Systems \& Health, 2002, pp.7-46. 